\title{
Transverse oscillations of systems of coronal loops
}

\author{
M. Luna ${ }^{1}$, J. Terradas ${ }^{2}$, R. Oliver ${ }^{1}$, and J.L. Ballester ${ }^{1}$
}

\begin{abstract}
We study the collective kinklike normal modes of a system of several cylindrical loops using the T-matrix theory. Loops that have similar kink frequencies oscillate collectively with a frequency which is slightly different from that of the individual kink mode. On the other hand, if the kink frequency of a loop is different from that of the others, it oscillates individually with its own frequency. Since the individual kink frequency depends on the loop density but not on its radius for typical $1 \mathrm{MK}$ coronal loops, a coupling between kink oscillations of neighboring loops take place when they have similar densities. The relevance of these results in the interpretation of the oscillations studied by Schrijver and Brown (2000) and Verwichte et al. (2004), in which transverse collective loop oscillations seem to be detected, is discussed. In the first case, two loops oscillating in antiphase are observed; interpreting this motion as a collective kink mode suggests that their densities are roughly equal. In the second case, there are almost three groups of tubes that oscillate with similar periods and therefore their dynamics can be collective, which again seems to indicate that the loops of each group share a similar density. All the other loops seem to oscillate individually and their densities can be different from the rest.
\end{abstract}

Subject headings: Sun: corona-magnetohydrodynamics (MHD)-waves-scattering

\section{Introduction}

Transverse coronal loop oscillations were discovered by the Transition Region and Coronal Explorer (TRACE) in 1998 (see, e.g. Aschwanden et al. 1999, 2002; Nakariakov et al. 1999). These oscillations were initiated shortly after a solar flare that disturbed the loops.

\footnotetext{
${ }^{1}$ Departament de Física, Universitat de les Illes Balears, 07122 Palma de Mallorca, Spain. Email: manuel.luna@uib.es, jaume.terradas@uib.es, ramon.oliver@uib.es and joseluis.ballester@uib.es

${ }^{2}$ Centre for Plasma Astrophysics, Katholieke Universiteit Leuven, Celestijnenlaan 200B, B-3001 Leuven, Belgium
} 
Since their first observation, transverse oscillations have been routinely observed and studied. Much before TRACE observations, the theory of loop oscillations was developed (Spruit 1981; Edwin and Roberts 1983; Cally 1986) and the different kinds of oscillations were studied. The observed transverse motions have been interpreted in terms of the fundamental kink mode of the fast magnetohydrodynamic (MHD) oscillation (Nakariakov et al. 1999), which is the only mode that can produce the observed transverse loop displacement.

In many cases the observed coronal loops belong to complex active regions and are not isolated but forming bundles or arcades of loops. For example, in Schrijver and Brown (2000) antiphase transverse oscillations of adjacent loops were reported. In addition, in Verwichte et al. (2004) phase and antiphase motions were observed in a post-flare arcade. On the other hand, it is currently debated whether active region coronal loops are monolithic or multistranded (see, e.g. Aschwanden et al. 2005; Klimchuk 2006; DeForest 2007). In the multistranded model, it is suggested that loops are formed by several tens or hundreds of strands considered as miniloops for which the heating plasma properties are approximately uniform in the transverse direction (Klimchuk 2006). Most analytical studies about transverse loop oscillations have only considered the properties of individual loops. However, from the information provided by the observations, it is necessary to study not only individual loops but also how several tubes can oscillate as a whole, since their joint dynamics can be different from that of a single loop. Only a few works have considered composite structures. Berton and Hevvaerts (1987) studied the MHD normal modes of a periodic magnetic medium. Murawski (1993) and Murawski and Roberts (1994) studied numerically the propagation of fast waves in two slabs unbounded in the longitudinal direction. In Díaz et al. (2005) the oscillations of the prominence thread structure were investigated. These authors found that in a system of equal fibrils the only non-leaky mode is the symmetric one, which means that all the fibrils oscillate in spatial phase with the same frequency. Luna et al. (2006) studied a system of two coronal slabs and found that the symmetric and antisymmetric modes can be trapped. A more complex system of two coronal cylinders was studied in Luna et al. (2008). Four trapped normal modes were found and the interchange of energy between loops was shown by solving the time-dependent problem. In Terradas et al. (2008) a multistranded loop formed by ten strands was considered. The composite loop oscillates transversely as a whole with a global motion of the strands after an external disturbance. This work shows that the bundle of strands oscillates with a combination of collective modes. On the other hand, an analytical approximation to the normal modes of a loop pair has been carried out by Van Doorsselaere et al. (2008). The authors assume the long wavelength approximation and obtain an analytical dispersion relation for two different tubes together with the four kink mode polarizations described in Luna et al. (2008).

In this work we aim to study the normal modes of a loop set with different physical and 
geometrical properties by using the scattering theory. The scattering theory, or its matricial formulation called T-matrix theory (see, e.g. Waterman and Truell 1961; Waterman 1969; Ramm 1986), was first applied to magnetic tubes by Bogdan and Zweibel (1985). These authors studied the interaction of acoustic plane waves with an ensemble of parallel magnetic fibrils distributed uniformly in the so-called spaghetti sunspot model. The authors derived and solved the dispersion relation in the long wavelength limit. In Bogdan and Cattaneo (1989) the frequency shifts and velocity eigenfunctions were calculated for the case of random fibril distributions of up to 100 flux tubes. Many other papers were published studying the cross section of a fibril spot insonified by external acoustic waves (see Bogdan and Fox 1991; Keppens et al. 1994). In all these papers a non-magnetized external medium was considered and the eigenfrequencies and eigenmodes of the acoustic oscillations were obtained.

In this paper we generalize the method to a system with an external magnetized medium, in order to extend previous works to coronal loop conditions. Our model consists of an ensemble of parallel cylinders, without gravity and curvature. We consider uniform magnetic field in the internal loop medium and in the external or coronal medium. This assumption allows the interaction of the tubes through fast MHD waves. In addition, we explicitly calculate the eigenvalues and eigenfunctions of the normal modes of the model.

This paper is organized as follows. In $\$ 2$ the loop ensemble model and the equations for its dynamics are presented. In $\oint 3$ we briefly describe the T-matrix theory and apply it to our model. With this theory the exact eigenfrequencies and eigenmodes of two non-identical loops are investigated in 4 . We study the dependence of the interaction with the relative density and radii of the loops. The study of three identical aligned loops is presented in $\$ 5$, In the same section the interaction between three non-identical loops is considered. Finally in $\$ 6$ the results are summarized and the main conclusions are drawn.

\section{Theoretical model}

The equilibrium configuration used to model the loop set is a system of $N$-cylindrical, parallel homogeneous flux tubes, with the $z$-axis pointing in the direction of the loop axes. All loops have the same length, $L$, and each individual loop, labeled $j$, is characterized by the position of its center in the $x y$-plane, $\mathbf{r}_{\mathrm{j}}=x_{\mathrm{j}} \mathbf{e}_{x}+y_{\mathrm{j}} \mathbf{e}_{y}$, its radius, $a_{\mathrm{j}}$, and its density, $\rho_{\mathrm{j}}$. The density of the coronal environment is $\rho_{0}$. The tubes and the external medium are permeated by a uniform magnetic field along the $z$-direction $\left(\mathbf{B}_{0}=B_{0} \mathbf{e}_{\mathrm{z}}\right)$. The Alfvén speed, $v_{\mathrm{A}}=B_{0} / \sqrt{\mu \rho}$, takes the value $v_{\mathrm{Aj}}$ inside the $j$-th loop and $v_{\mathrm{A} 0}$ in the surrounding corona $\left(v_{\mathrm{Aj}}<v_{\mathrm{A} 0}\right)$. 
Linear perturbations about this equilibrium for a perfectly conducting fluid can be readily described using the ideal MHD equations. In the zero- $\beta$ limit these equations can be written as

$$
\left(\frac{\partial^{2}}{\partial t^{2}}-v_{\mathrm{A}}^{2} \nabla^{2}\right) p_{\mathrm{T}}=0,
$$

where $p_{\mathrm{T}}$ is the total pressure perturbation

$$
p_{\mathrm{T}}=\frac{B_{0}}{\mu} B_{z}
$$

and $B_{z}$ is the $z$-component of the magnetic field perturbation. The other perturbed quantities, namely the velocity, $\mathbf{v}$, the magnetic field perturbation, $\mathbf{B}$, and the density perturbation, $\rho$, can be derived from $p_{\mathrm{T}}$. We have assumed a $z$-dependence of the perturbations of the form $e^{-i k_{z} z}$. The line-tying effect is incorporated by setting $k_{z}=q \pi / L$ where $q$ is an integer number. Hereafter we concentrate on the fundamental mode and take $q=1$. We only consider problems for which the time dependence is a simple harmonic oscillation with frequency $\omega$. Then, the total pressure perturbation can be written in cylindrical coordinates as

$$
p_{\mathrm{T}}=e^{i\left(k_{z} z-\omega t\right)} \psi(r, \varphi)
$$

where $\psi(r, \varphi)$ is a function that includes the dependence on $r$ and $\varphi$. Inserting this expression in equation (1), we obtain the scalar Helmholtz equation

$$
\nabla_{\perp}^{2} \psi+k_{\perp}^{2} \psi=0
$$

where $\perp$ refers to the direction perpendicular to the magnetic field $\mathbf{B}_{0}$, i.e. to the $z$-axis, and $k_{\perp}$ is

$$
k_{\perp}^{2}=\frac{\omega^{2}-k_{z}^{2} v_{\mathrm{A}}^{2}}{v_{\mathrm{A}}^{2}} .
$$

Hereafter, the $\perp$ symbol is dropped for the sake of simplicity.

\section{Normal modes}

The scattering theory, or its matricial formulation called T-matrix theory, provides an scheme to find analytically the normal modes of a system of scatterers in which waves are described by a Helmholtz equation (Ramm 1986). We fulfill the T-matrix theory requirements because our ensemble of $N$ loops can be considered a collection of scatterers and the perturbed total pressure is described by equation (4). 
The T-matrix scheme states that the $j$-th flux tube generates an outgoing scattered wave, $\psi_{\mathrm{sc}}^{\mathrm{j}}$, in a field position $\mathbf{r}$ (in the two-dimensional $x y$-plane) that adds to the waves scattered from the other loops to produce the following net external field (Bogdan and Cattaneo 1989)

$$
\psi(\mathbf{r})=\sum_{\mathrm{j}}^{\mathrm{N}} \psi_{\mathrm{sc}}^{\mathrm{j}}\left(\mathbf{r}-\mathbf{r}_{\mathrm{j}}\right) .
$$

The scattered wave by the $j$-th loop is produced as a response of an exciting wave produced by the external field minus the contribution of the mentioned loop,

$$
\psi_{\mathrm{ex}}^{\mathrm{j}}\left(\mathbf{r}-\mathbf{r}_{\mathrm{j}}\right)=\psi(\mathbf{r})-\psi_{\mathrm{sc}}^{\mathrm{j}}\left(\mathbf{r}-\mathbf{r}_{\mathrm{j}}\right) .
$$

With equations (6) and (7) the exciting field, $\psi_{\mathrm{ex}}^{\mathrm{j}}$, may be written entirely in terms of the scattered field, resulting in the self-consistency field equation (Bogdan and Cattaneo 1989). This system of equations may then be closed by noting that the exciting and scattered fields are further related by linear operators, $\mathbf{T}^{\mathrm{j}}$, that describe the scattering properties of the individual flux tubes (Bogdan and Cattaneo 1989; Waterman 1969; Ramm 1986)

$$
\psi_{\mathrm{sc}}^{\mathrm{j}}\left(\mathbf{r}-\mathbf{r}_{\mathrm{j}}\right)=\mathbf{T}^{\mathrm{j}} \psi_{\mathrm{ex}}^{\mathrm{j}}\left(\mathbf{r}-\mathbf{r}_{\mathrm{j}}\right) .
$$

The key point is that the linear operators $\mathbf{T}^{\mathrm{j}}$ depend exclusively on the individual loop and external medium properties and can be directly computed through the boundary conditions on the loop-external medium interphase as we will see below.

The external field to the $j$-th loop can be decomposed with equation (7) as an excitation field on this loop and a scattered field by this loop. The excitation field has no sources in the $j$-th loop, i.e. it is the scattered field of the other loops, so it can be written as

$$
\psi_{\mathrm{ex}}^{\mathrm{j}}\left(R_{\mathrm{j}}, \varphi_{\mathrm{j}}\right)=\sum_{\mathrm{m}=-\infty}^{\infty} \alpha_{\mathrm{m}}^{\mathrm{j}} J_{\mathrm{m}}\left(k_{0} R_{\mathrm{j}}\right) e^{i \mathrm{~m} \varphi_{\mathrm{j}}},
$$

where $\alpha_{\mathrm{m}}^{\mathrm{j}}$ are the expansion coefficients of order $m$, that depend on the $k_{z}$ wave number and the frequency $\omega$, and $R_{\mathrm{j}}$ and $\varphi_{\mathrm{j}}$ are the local polar coordinates centered at $\mathbf{r}_{\mathrm{j}}$, defined through $R_{\mathrm{j}}=\left|\mathbf{r}-\mathbf{r}_{\mathrm{j}}\right|$ and $\cos \varphi_{\mathrm{j}}=\mathbf{e}_{x} \cdot\left(\mathbf{r}-\mathbf{r}_{\mathrm{j}}\right) /\left|\mathbf{r}-\mathbf{r}_{\mathrm{j}}\right|$. Here $J_{\mathrm{m}}$ is the Bessel function of the first kind and order $m$ and $k_{0}$ is $k$ in the external medium calculated using equation (5). With equations (8) and (9), we find the scattered field in terms of an outgoing wave with sources at $\mathbf{r}_{\mathrm{j}}$,

$$
\psi_{\mathrm{sc}}^{\mathrm{j}}\left(R_{\mathrm{j}}, \varphi_{\mathrm{j}}\right)=\sum_{\mathrm{m}=-\infty}^{\infty} T_{\mathrm{mm}}^{\mathrm{j}} \alpha_{\mathrm{m}}^{\mathrm{j}} H_{\mathrm{m}}^{(1)}\left(k_{0} R_{\mathrm{j}}\right) e^{i \mathrm{~m} \varphi_{\mathrm{j}}},
$$

where $T_{\mathrm{mm}}^{\mathrm{j}}$ are the matrix diagonal elements of the operator $\mathbf{T}^{\mathrm{j}}$ projected on the local basis, called T-matrix. The non-diagonal elements of this matrix are zero for axisymmetric tubes 
(Bogdan and Zweibel 1985). The functions $H_{\mathrm{m}}^{(1)}$ are the Hankel functions of the first kind. With equations (6), (17), (9), and (10), we find the following expression for the total field

$$
\psi(\mathbf{r})=\sum_{\mathrm{m}=-\infty}^{\infty} \alpha_{\mathrm{m}}^{\mathrm{j}}\left[J_{\mathrm{m}}\left(k_{0} R_{\mathrm{j}}\right)+T_{\mathrm{mm}}^{\mathrm{j}} H_{\mathrm{m}}^{(1)}\left(k_{0} R_{\mathrm{j}}\right)\right] e^{i \mathrm{~m} \varphi_{\mathrm{j}}},
$$

in which the external field to $j$-th loop is decomposed as an excitation on this loop and a scattered field by this loop (Waterman 1969).

Following the development of Bogdan and Cattaneo (1989), a linear algebraic system of equations for the complex coefficients $\alpha_{\mathrm{m}}^{\mathrm{j}}$ may then be obtained. We first substitute equation (6) in equation (17) in order to obtain the self-consistency requirement

$$
\psi_{\mathrm{ex}}^{\mathrm{j}}\left(\mathbf{r}-\mathbf{r}_{\mathrm{j}}\right)=\sum_{\mathrm{i} \neq \mathrm{j}}^{\mathrm{N}} \psi_{\mathrm{sc}}^{\mathrm{i}}\left(\mathbf{r}-\mathbf{r}_{\mathrm{i}}\right)
$$

Next the exciting and scattered fields are replaced by their basis expansions, equations (9) and (10), and the translation formula (see appendix of Bogdan and Cattaneo 1989) is used to express the scattered wave centered in the $i$-th loop into an excitation at $j$-th flux tube. Finally, we obtain the following set of equations

$$
\alpha_{\mathrm{m}}^{\mathrm{j}}+\sum_{\mathrm{i} \neq \mathrm{j}}^{\mathrm{N}} \sum_{\mathrm{n}=-\infty}^{\infty} \alpha_{\mathrm{n}}^{\mathrm{i}} T_{\mathrm{nn}}^{\mathrm{i}} H_{\mathrm{n}-\mathrm{m}}^{(1)}\left(\mathrm{k}_{0}\left|\mathbf{r}_{\mathrm{j}}-\mathbf{r}_{\mathrm{i}}\right|\right) e^{i(\mathrm{n}-\mathrm{m}) \varphi_{\mathrm{ji}}}=0,
$$

where $\varphi_{\mathrm{j}}$ is the angle formed by the center of the $i$-th loop with respect to the center of the $j$-th flux tube. As we will see below with this equation we can find the $\alpha_{\mathrm{m}}^{\mathrm{j}}$ coefficients and the frequencies $\omega$, from which the spatial structure of the normal modes can be determined. From equation (13), we see that the expansion coefficient of order $m$ of the $j$-th loop, $\alpha_{\mathrm{m}}^{\mathrm{j}}$, is coupled to all expansion coefficients of the other loops. This fact reflects the collective nature of the normal modes. With the $\alpha_{\mathrm{m}}^{\mathrm{j}}$ and equations (6) and (10) we find the net external field.

The internal or transmitted field is

$$
\psi_{\mathrm{tr}}^{\mathrm{j}}\left(\mathbf{r}-\mathbf{r}_{\mathrm{j}}\right)=\sum_{\mathrm{m}=-\infty}^{\infty} \beta_{\mathrm{m}}^{\mathrm{j}} J_{\mathrm{m}}\left(k_{\mathrm{j}} R_{\mathrm{j}}\right) e^{i \mathrm{~m} \varphi_{\mathrm{j}}}
$$

where $k_{\mathrm{j}}$ is the transverse wavenumber inside the $j$-th loop calculated using equation (5). The Bessel functions of the second kind, $Y_{\mathrm{m}}$, are not considered in the expansion of the internal field (eq. [14) because they are singular at the loop axes. The transmitted field (eq. [14) can be calculated through the boundary conditions, namely the continuity of the total pressure 
perturbation (2) and the radial component of the velocity at $R_{\mathrm{j}}=a_{\mathrm{j}}$ (see Goedbloed 1983). In terms of the $\psi$ fields they are expressed as follows

$$
\begin{aligned}
\left.\psi_{\mathrm{tr}}^{\mathrm{j}}\left(k_{\mathrm{j}} R_{\mathrm{j}}\right)\right|_{R_{\mathrm{j}}=a_{\mathrm{j}}} & =\left.\psi\left(k_{0} R_{\mathrm{j}}\right)\right|_{R_{\mathrm{j}}=a_{\mathrm{j}}}, \\
\frac{\left.\psi_{\mathrm{tr}}^{\mathrm{j} \prime}\left(k_{\mathrm{j}} R_{\mathrm{j}}\right)\right|_{R_{\mathrm{j}}=a_{\mathrm{j}}}}{k_{\mathrm{j}}} & =\frac{\left.\psi^{\prime}\left(k_{0} R_{\mathrm{j}}\right)\right|_{R_{\mathrm{j}}=a_{\mathrm{j}}}}{k_{0}}
\end{aligned}
$$

where the prime is the derivative with respect to the function argument, $\psi^{\prime}(x)=\partial \psi(x) / \partial x$.

Equation (13) is completely general for a system of $N$ cylindrical flux tubes (see Keppens 1995, for non-axisymmetric expressions) and all the information of the individual loops is included in the T-matrix elements, $T_{\mathrm{mm}}^{\mathrm{j}}$. These elements are calculated through the boundary conditions at the interphase between the loop and the external medium. With equations (11), (14), (15), and (16) we find the $T_{\mathrm{mm}}^{\mathrm{j}}$ element expression

$$
T_{\mathrm{mm}}^{\mathrm{j}}=\frac{k_{\mathrm{j}}{ }^{2} k_{0} J_{\mathrm{m}}\left(k_{\mathrm{j}} a_{\mathrm{j}}\right) J^{\prime}{ }_{\mathrm{m}}\left(k_{0} a_{\mathrm{j}}\right)-{k_{0}}^{2} k_{\mathrm{j}}{J^{\prime}}_{\mathrm{m}}\left(k_{\mathrm{j}} a_{\mathrm{j}}\right) J_{\mathrm{m}}\left(k_{0} a_{\mathrm{j}}\right)}{k_{0}{ }^{2} k_{\mathrm{j}} H_{\mathrm{m}}^{(1)}\left(k_{0} a_{\mathrm{j}}\right) J^{\prime}{ }_{\mathrm{m}}\left(k_{\mathrm{j}} a_{\mathrm{j}}\right)-k_{\mathrm{j}}{ }^{2} k_{0}{H^{\prime}}_{\mathrm{m}}^{(1)}\left(k_{0} a_{\mathrm{j}}\right) J_{\mathrm{m}}\left(k_{\mathrm{j}} a_{\mathrm{j}}\right)} .
$$

Equation (17) is the generalization of Bogdan and Zweibel (1987); Bogdan and Cattaneo (1989) to the case of a magnetized environment. The zeroes of the denominator correspond to the dispersion relation of the individual loop (see, e.g. Cally 1986).

Finally, note that with the boundary conditions (eqs. [15] and [16]) it is possible to calculate the $\beta_{\mathrm{m}}^{\mathrm{j}}$ coefficients

$$
\beta_{\mathrm{m}}^{\mathrm{j}}=\frac{J_{\mathrm{m}}\left(k_{0} a_{\mathrm{j}}\right)+T_{\mathrm{mm}}^{\mathrm{j}} H_{\mathrm{m}}^{(1)}\left(k_{0} a_{\mathrm{j}}\right)}{J_{\mathrm{m}}\left(k_{\mathrm{j}} a_{\mathrm{j}}\right)} \alpha_{\mathrm{m}}^{\mathrm{j}},
$$

which can be inserted into equation (14) to obtain the internal field, $\psi_{\mathrm{tr}}^{\mathrm{j}}$.

Equation (13) is formally an infinite system of equations for an infinite number of unknowns $\left(\alpha_{\mathrm{m}}^{\mathrm{j}}\right)$. In order to solve it, we truncate the system into a finite number of equations and unknowns by setting $\alpha_{\mathrm{m}_{\mathrm{t}}+1}^{\mathrm{j}}=0$ for azimuthal numbers greater than a truncation number $\left(m>m_{\mathrm{t}}\right)$. To ensure the convergence of solutions, they must be independent of the truncation number $m_{\mathrm{t}}$. With these considerations, the solution of equation (13) reduces to solve a homogeneous linear system of $N\left(2 m_{\mathrm{t}}+1\right)$ equations and $N\left(2 m_{\mathrm{t}}+1\right)$ unknowns. For this system of equations to have a non-trivial solution, its determinant must be zero. This requirement gives the dispersion relation as a transcendent equation. We solve the dispersion relation numerically and find the frequencies of the normal modes and with these frequencies we calculate the $\alpha_{\mathrm{m}}^{\mathrm{j}}$ expansion coefficients. With equations (6) and (14) we find the net field in the external medium and the transmitted field in each loop. In all our calculations, 
solutions are independent of the truncation number for values $m_{\mathrm{t}}>5$ but we fix this number to $m_{\mathrm{t}}=20$ to more confidently ensure their convergence. With the method presented here we have obtained the results of the following sections.

\section{Interaction between two loops}

First, we compute the normal modes of two non-identical loops with the T-matrix theory outlined in 93 . In this section we study the dependence of the interaction as a function of the density and radii of the loops. We consider a system of two loops with radii $a_{1}=a_{2}=a=0.03 L$ and separated a distance $d=3 a$. The first loop density is $\rho_{1}=3 \rho_{0}$ while $\rho_{2}$ is changed from $\rho_{2}=\rho_{0}$ to $5 \rho_{0}$ to study its influence on the normal mode properties. We concentrate on the kinklike modes in which the individual loops move more or less as kink and suffer the largest transverse displacement. There are other higher order normal modes whose spatial structure is more complex, i.e. fluting modes. We find four kinklike normal modes named $P_{x}, A P_{y}, P_{y}$, and $A P_{x}$, where $P$ and $A P$ refer to phase or antiphase motions of the loops, respectively, and the subscript $x$ or $y$ refers to the direction of the motion along the $x$ - or $y$-axes. The frequencies of oscillation of these four modes as a function of $\rho_{2} / \rho_{0}$ are displayed in Figure 1. The bottom solid line is associated to the $P_{x}$ and $A P_{y}$ modes, which almost have the same frequency (see inbox Fig. 1). The same behavior is found for the top solid line, which corresponds to the $P_{y}$ and $A P_{x}$ modes. We see that the collective normal modes (solid lines) do not coincide with the kink frequencies of the individual loops (dashed lines), a discrepancy caused by the interaction between loops. This interaction is maximal when $\rho_{2}=\rho_{1}$ (dotted line), and the normal modes $P_{x}, A P_{y}, P_{y}$, and $A P_{x}$ become the modes $S_{x}, A_{y}, S_{y}$, and $A_{y}$ reported in Luna et al. (2008). The opposite situation takes place when $\rho_{2}$ is sufficiently different from $\rho_{1}$ : the collective frequencies are closer to the individual kink frequencies and the system behaves as a pair of independently oscillating loops. In this regime, the $P_{x}$ and $A P_{y}$ modes correspond to the individual oscillations of the denser

loop in the $x$ - and $y$-direction respectively and possess identical frequencies, whereas the $P_{y}$ and $A P_{x}$ modes are the individual oscillations of the rarer loop in the $x$ - and $y$-direction, respectively, and also share the same frequency. Figure 1 can be interpreted globally as an avoided crossing of the kink modes of the loops: far from the coupling, each branch is associated to the individual loop kink mode, but near the avoided crossing motions are associated to the two loops to produce four collective kinklike modes. As for as kinklike solutions are concerned, loops interact for densities approximately in the range $\rho_{2}=2 \rho_{0}$ to $4 \rho_{0}$.

The total pressure perturbation of the $P_{x}$ and $A P_{x}$ modes is plotted in Figure 2 for two 


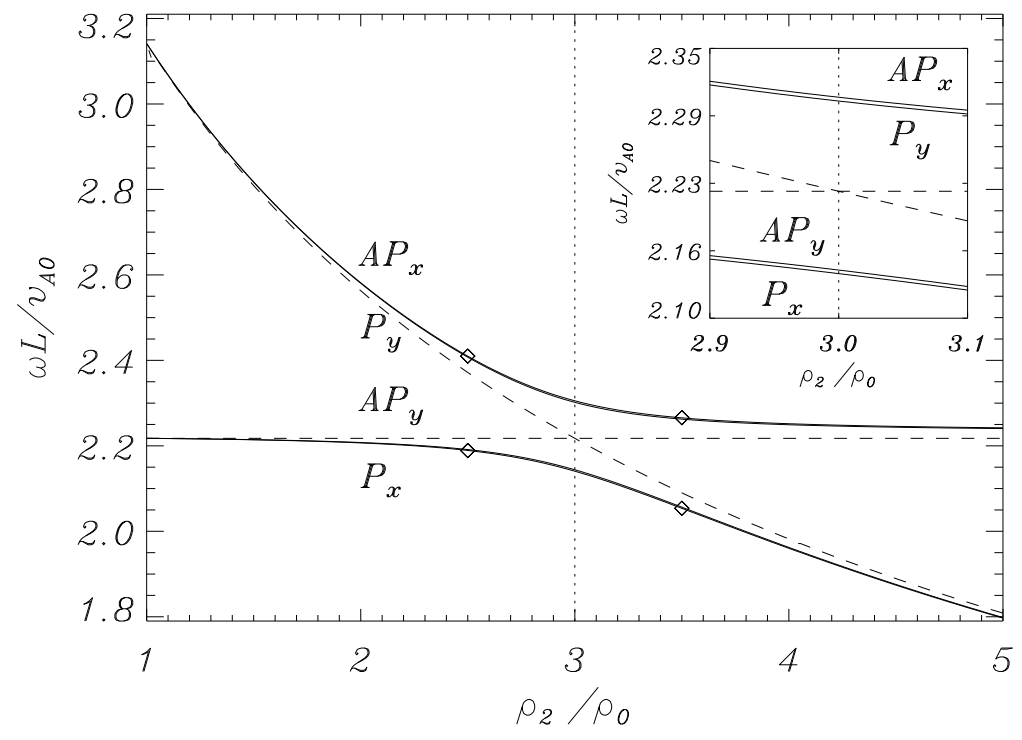

Fig. 1.- Dimensionless frequency, $\omega L / v_{\mathrm{A} 0}$, as a function of the internal density of the second loop. The bottom solid line is associated to the two kinklike normal modes $P_{x}$ and $A P_{y}$, which have very similar frequencies. In the same way, the upper solid line is associated to the $P_{y}$ and $A P_{x}$ modes. In the inner plot a detailed view for $\rho_{2} \approx \rho_{1}$ is displayed showing that the solid lines are in fact double lines. The two dashed lines are the individual kink frequencies of each loop. We see that the kink frequency of loop 1 is constant and the frequency of loop 2 decreases with $\rho_{2}$, because $\rho_{1}$ is constant and $\rho_{2}$ changes. The vertical dotted line is plotted at $\rho_{2}=\rho_{1}$. Diamonds mark the frequencies of the modes represented in Fig. 2,

cases in which the loop interaction is important $\left(\rho_{2}=2.5 \rho_{0}\right.$ and $\left.3.5 \rho_{0}\right)$. The behavior of the other two modes, $A P_{y}$ and $P_{y}$, is analogous to that of the $P_{x}$ and $A P_{x}$ modes and thus their spatial structure is not shown. In contrast to the case $\rho_{1}=\rho_{2}$, in which the interaction is maximal and thus the two loops oscillate with equal amplitudes (see Fig. 2 of Luna et al. 2008) the solutions in Figure 2 display an imbalance in the oscillatory amplitude of the two loops. The largest amplitude of the pressure perturbation corresponds to the denser loop for the $P_{x}$ mode (see Figs. 2a and $2 \mathrm{~b}$ ), while it occurs in the rarer loop for the $A P_{x}$ mode (see Figs. 2 c and $2 \mathrm{~d})$.

Secondly, we consider the same system of two loops but now the densities are fixed to $\rho_{1}=\rho_{2}=3 \rho_{0}$, the radius of the left loop is $a_{1}=0.03 L$, and the right loop radius, $a_{2}$, is allowed to vary. The distance between the tube centers is $3 a_{\mathrm{M}}$, where $a_{\mathrm{M}}$ is the averaged radius defined as $a_{\mathrm{M}}=\left(a_{1}+a_{2}\right) / 2$. With this condition the separation measured 

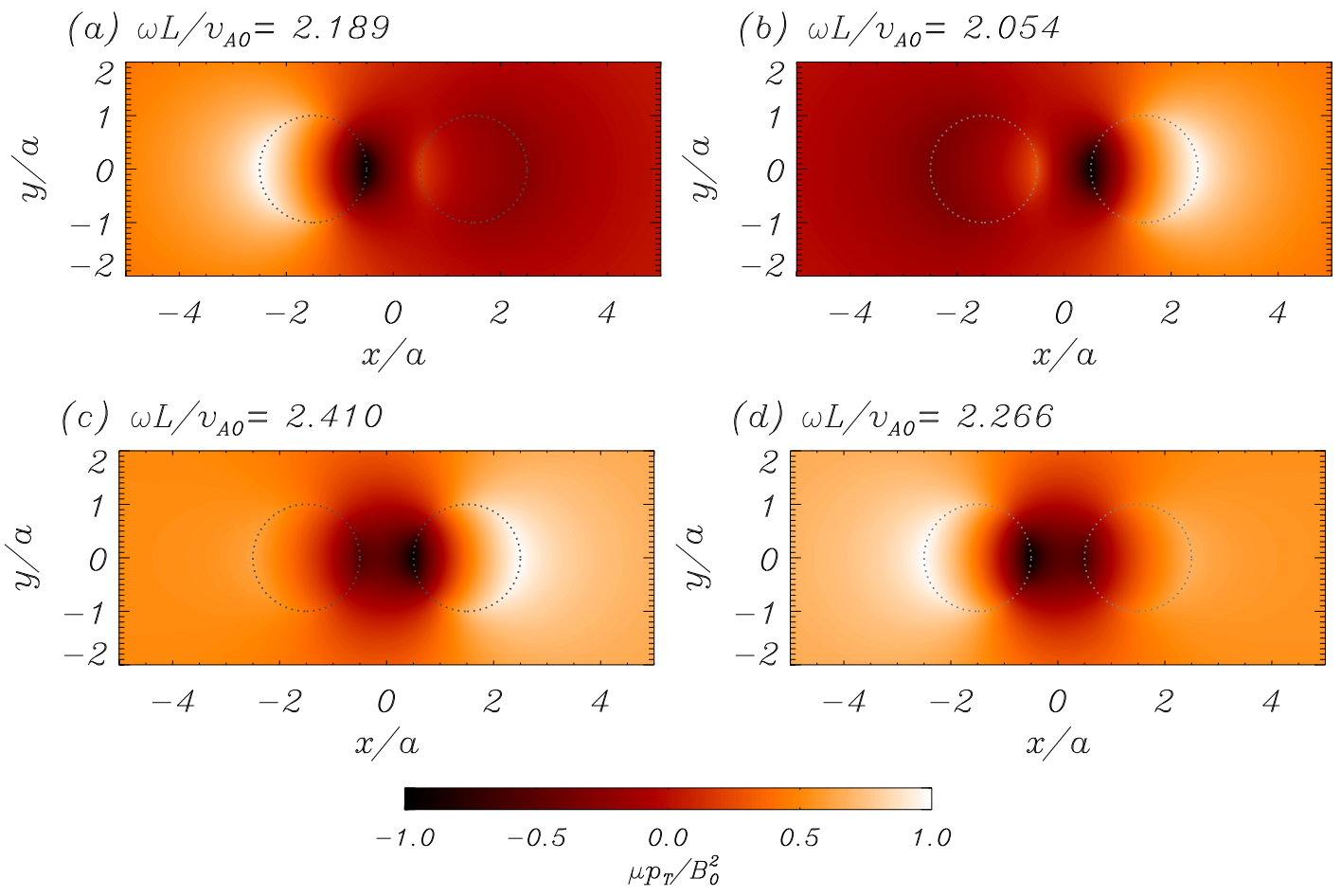

Fig. 2.- Total pressure perturbation of the fast collective normal modes $P_{x}$ and $A P_{x}$ (plotted in the $x y$-plane) for a fixed density of the left loop $\left(\rho_{1}=3 \rho_{0}\right)$ and different densities of the right loop $\left(\rho_{2}\right)$. The panels show the $P_{x}$ mode with (a) $\rho_{2}=2.5 \rho_{0}$ and (b) $\rho_{2}=3.5 \rho_{0}$; the $A P_{x}$ mode with (c) $\rho_{2}=2.5 \rho_{0}$ and (d) $\rho_{2}=3.5 \rho_{0}$. The frequencies of the modes are given on top of the corresponding panels. The dotted lines show the boundaries of the unperturbed tubes. Regions of the positive (negative) perturbed total pressure represent density enhancements (decrements), so that in (a) and (b) the loops move in phase in the $x$-direction, while in $(\mathbf{c})$ and $(\mathbf{d})$ they move in antiphase in the $x$-direction.

in averaged radius units is constant. The frequencies of the four modes $P_{x}, A P_{y}, P_{y}$, and $A P_{x}$ are plotted in Figure 3. As in Figure 1 the collective frequencies (solid lines) are different from the individual kink frequencies (dashed lines), showing the collective nature of the oscillations. The chosen range of radii are those measured in TRACE observation of transverse oscillations (see, e.g. Aschwanden et al. 2003). In Figure 3 we see that the collective frequencies are more or less constant; moreover the amplitude of the oscillation is more or less equal in each tube. Then, in the considered range of radii the interaction 
between kink modes is strong and does not significantly depend on the loop radii.

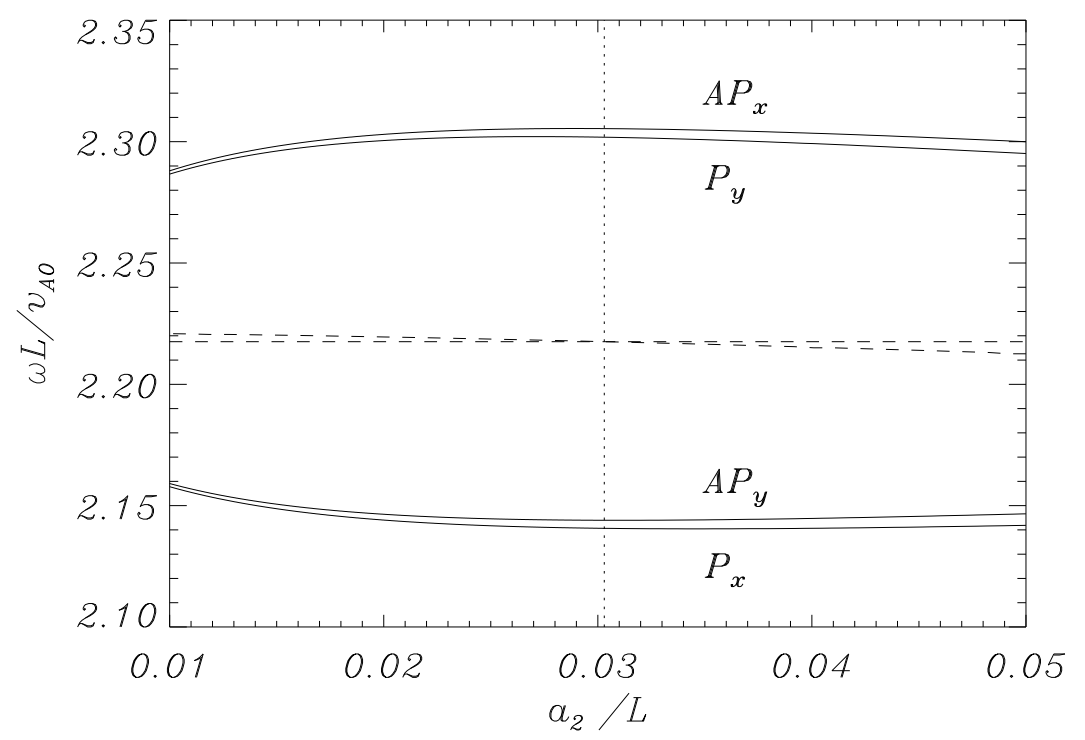

Fig. 3.- Dimensionless frequency, $\omega L / v_{\mathrm{A} 0}$, of the four collective kinklike modes $P_{x}, A P_{y}, P_{y}$, and $A P_{x}$ (solid lines), as a function of the normalized right loop radius, $a_{2} / L$. As in Fig. 1 the two individual kink frequencies are plotted (dashed lines), where the horizontal dashed line corresponds to the left loop and the one with a slight dependence on $a_{2}$ corresponds to the right loop.

\section{Interaction between three loops}

\subsection{Equal loop densities}

We first study the situation in which the density and radii of the loops is the same and find that there are eight kinklike normal modes, whose eigenfunctions are plotted in Figure 4 , with the modes ordered by increasing frequency. The lower frequency corresponds to a mode in which the three loops move in phase in the $x$-direction (Fig. $4 a$ ), whereas in the higher frequency mode (Fig. $4 \mathrm{~h}$ ) the three loops move in phase in the $y$-direction. This behavior is different from that of the system of two loops (see \$4), in which the higher frequency mode corresponds to the $A_{x}$ instead of the $S_{y}$ mode. The modes of Figures $4 \mathrm{a}$, $4 \mathrm{~b}$, $4 \mathrm{~g}$, and $4 \mathrm{~h}$ are kinklike while the other four modes of Figures 4 $\mathrm{c}$, 44d, 4e, and $4 \mathrm{f}$ combine kink and fluting oscillations: the two left and right loops oscillate with a kinklike motion whereas the central loop oscillates with a fluting motion. We also refer to these modes as kinklike because at least one loop oscillates with a kinklike behavior. In these modes the central loop contributes 
appreciably to the total field (eq.[10]) with the multipole $m=2$. Between the frequencies of the modes plotted in Figures $4 \mathrm{~d}$ and 4 e there are modes with the three loops oscillating with fluting motions and even with more complex structure associated to $m>2$ solutions. Then we call these modes flutinglike. They are not further analysed because they do not produce transverse displacements of the loops.

\subsection{Different loop densities}

Now we consider the dependence of the interaction on the loop density. The loop radii are fixed to $a_{1}=a_{2}=a_{3}=a=0.03 L$, the separation between adjacent loops is $d=3 a$, the densities of loops 1 and 2 are fixed to $\rho_{1}=3 \rho_{0}$ and $\rho_{2}=2 \rho_{0}$, and $\rho_{3}$ is changed from $\rho_{0}$ to $4 \rho_{0}$. Six kinklike normal modes, rather than eight, are found and their frequencies are plotted as a function of $\rho_{3}$ in Figure 5. There are six branches associated to the six kinklike modes, that have been labeled $m_{1}$ to $m_{6}$ starting with the lowest frequency mode. We have chosen $\rho_{1}$ and $\rho_{2}$ in such a way that loops 1 and 2 are basically decoupled (see \$4). Figure 5 is similar to Figure 1 and can be interpreted as two avoided crossings of the individual kink modes of the three loops. Far from the couplings, the loops behave independently. This fact is illustrated in Figure 6. In this figure we have plotted the modes associated to the branches $m_{1}, m_{3}$ and $m_{6}$ in the top, central, and bottom rows, respectively. The modes $m_{2}, m_{4}$ and

$m_{5}$ have an equivalent behavior, and have not been plotted. Far from the coupling region the $m_{1}$ and $m_{2}$ solutions are associated to the individual kink oscillations of the denser loop in the $x$ - and $y$-direction, respectively. In the same way, the branches $m_{3}$ and $m_{4}$ are associated to the individual kink mode of the intermediate density loop and the branches $m_{5}$ and $m_{6}$ to the individual kink oscillations of the rarest loop. On the other hand, at the couplings the loops interact by pairs as we see in Figure 7. The interacting pair oscillates with a collective normal mode whereas the other loop oscillates individually. In the first avoided crossing, for $\rho_{3}=\rho_{2}=2 \rho_{0}$, the branches $m_{3}$ and $m_{4}$ are coupled with $m_{5}$ and $m_{6}$ (see Fig. 5 ), associated to loops 2 and 3, that oscillate collectively as we see in Figures $7 \mathrm{c}$ and $7 \mathrm{k}$. The branches $m_{1}$ and $m_{2}$ are uncoupled and loop 1 oscillates independently from the other two, as we see in Figure $7 \mathrm{a}$. In the second avoided crossing at $\rho_{3}=\rho_{1}=3 \rho_{0}$ the branches $m_{1}$ and $m_{2}$ are coupled with $m_{3}$ and $m_{4}$, while $m_{5}$ and $m_{6}$ are independent. Therefore, in this avoided crossing the interaction is between loops 1 and 3 (Figs. 7b and 7 d) and loop 2 oscillates independently (Fig. 7f). It is important to note that the collective modes of the two coupled tubes have a different frequency ordering with respect to the case of two loops, studied in \$4, because of the presence of loop 1 .

Comparing Figures 1 and 5 the coupling regions occur in a narrower range of density 

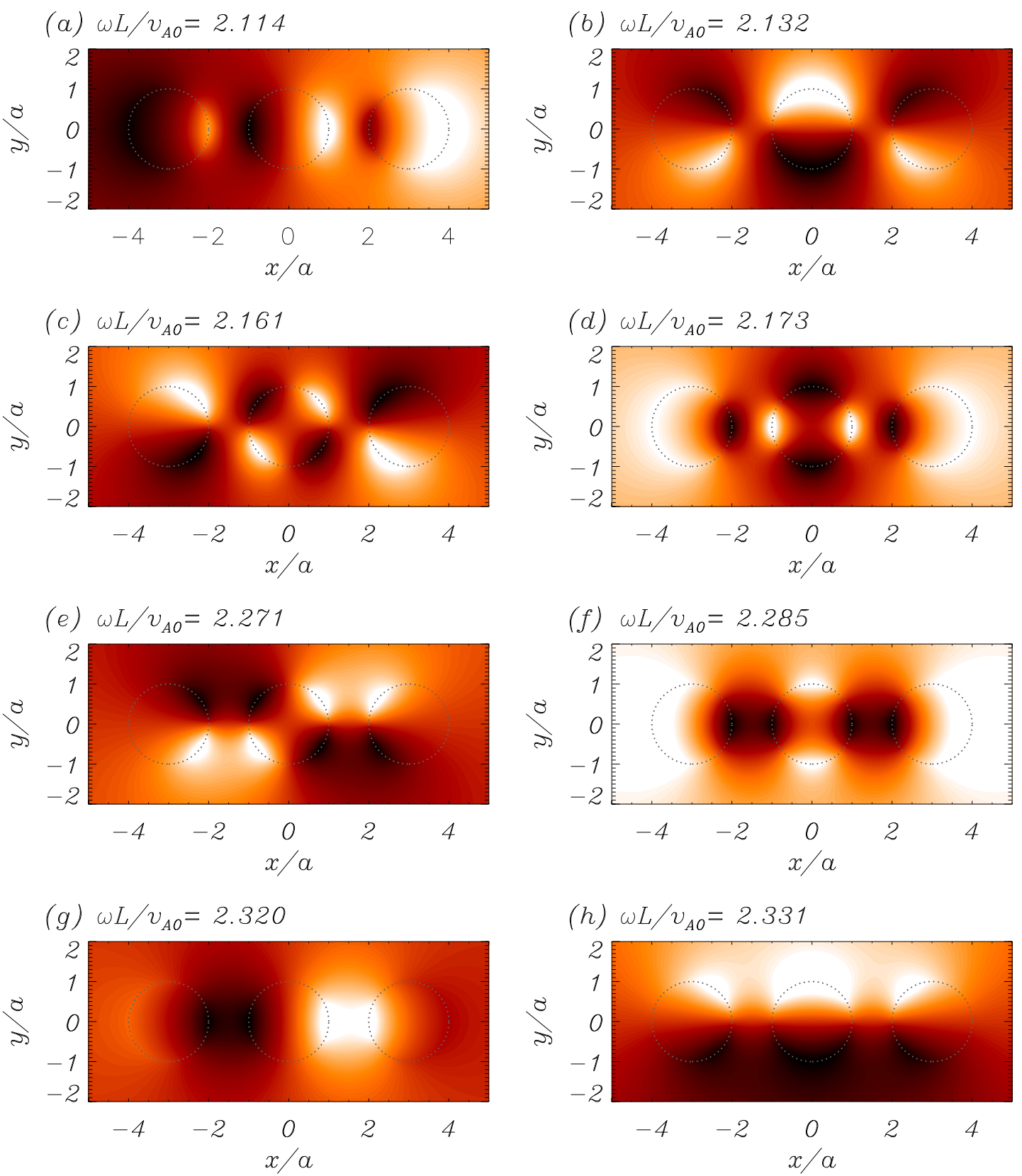

Fig. 4.- Total pressure perturbation of the eight kinklike collective normal modes of three identical loops. The densities are fixed to $\rho_{1}=\rho_{2}=\rho_{3}=3 \rho_{0}$, the radii to $a_{1}=a_{2}=a_{3}=$ $a=0.03 L$, and the separation between adjacent loops is $d=3 a$. 


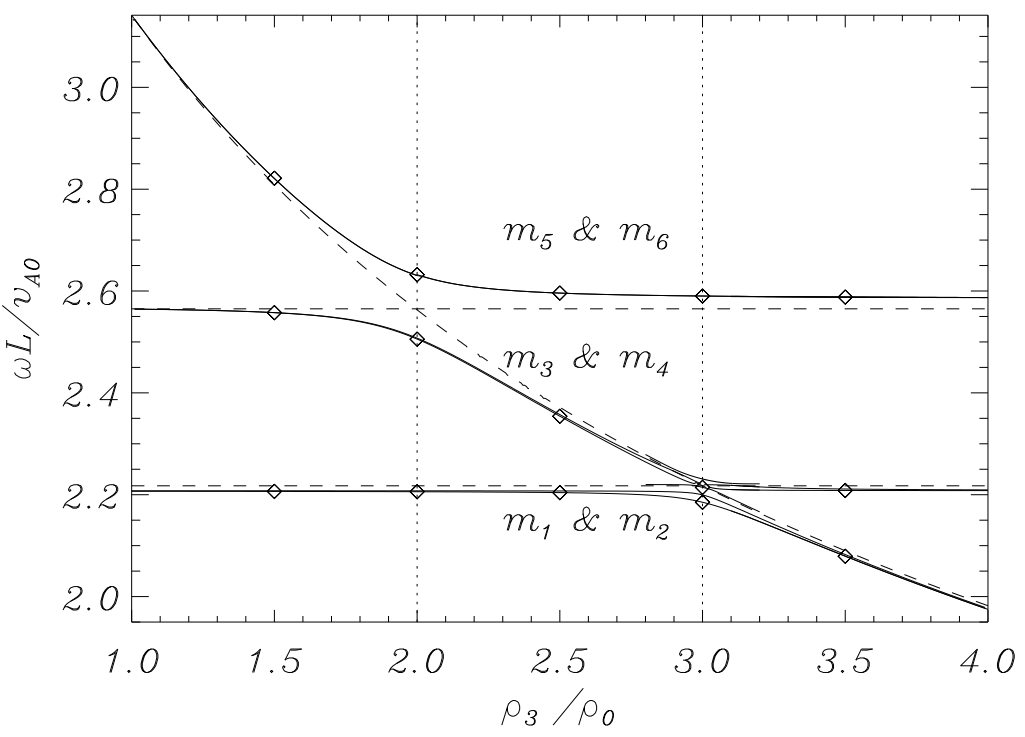

Fig. 5.- Same as Fig. 1 for the collective frequencies of three aligned loops plotted as a function of the density of loop 3. Solid lines correspond to the frequencies of the six collective kinklike modes. Dashed lines correspond to the individual kink frequencies of the loops. The horizontal bottom and upper dashed lines corresponds to the kink frequencies of loops 1 and 2 respectively. The other dashed curve corresponds to the kink frequency of the third loop, with variable density $\rho_{3}$. Diamonds mark the frequencies of the modes represented in Figs. 6 and 7.

values in the three loop system than for two tubes. The physical meaning is that only loops with similar densities are coupled in the three loop ensemble. In this system, it is important to note that in the second avoided crossing at $\rho_{3} \approx \rho_{1}$ loop 2 does not participate of the collective dynamics despite being the closest tube to the interacting loops.

The results discussed so far in this subsection correspond to different densities of loops 1 and 2. Nevertheless, if the densities of loops 1 and 2 are similar, their interaction is more important and the description of the dispersion diagram and the normal modes of the system is much more complex. In this case, there are eight kinklike normal modes. In this situation there are not only interactions between pairs of loops but also interactions between three loops. There are modes associated to the ensemble formed by tubes 1 and 2, individual oscillations of the cylinder 3 and the ensemble of the three loops depending on $\rho_{3}$. A particular case of this situation is the three identical loops previously discussed (\$5.1) in which all modes are associated to the collectivity. 

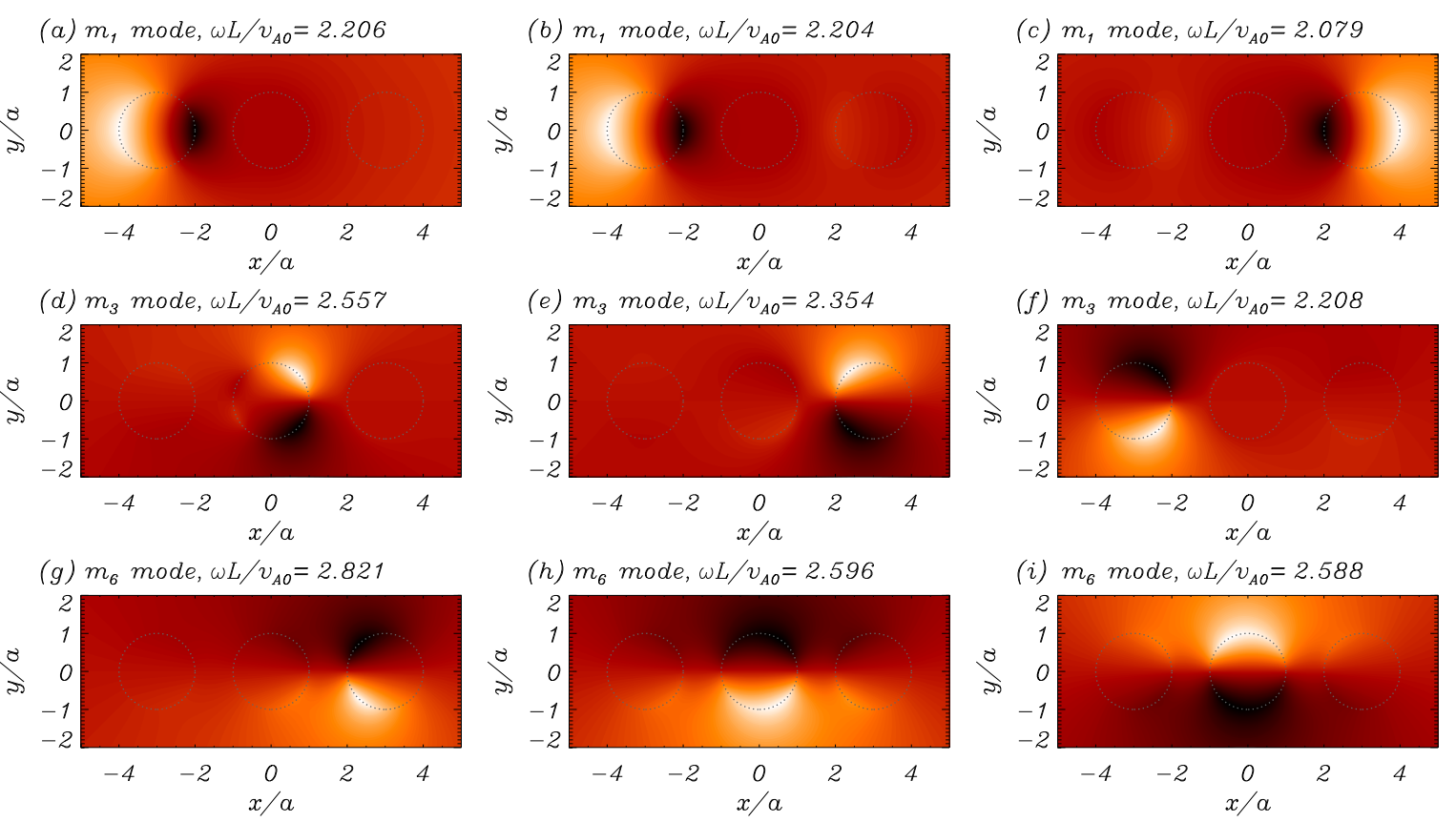

Fig. 6.- Same as Fig. 4 for three values of $\rho_{3}$ far from the two coupling regions of Fig. 5 , The densities of loops 1 and 2 are fixed to $\rho_{1}=3 \rho_{0}$ and $\rho_{2}=2 \rho_{0}$ and $\rho_{3}$ is changed. The loop radii are fixed to $a_{1}=a_{2}=a_{3}=0.03 \mathrm{~L}$. In the top, central, and bottom rows of panels the $m_{1}, m_{3}$, and $m_{6}$ modes are plotted for $(\mathbf{a}),(\mathbf{d})$, and $(\mathbf{g}) \rho_{3}=1.5 \rho_{0} ;(\mathbf{b}),(\mathbf{e})$, and $(\mathbf{h})$ $\rho_{3}=2.5 \rho_{0} ;(\mathbf{c}),(\mathbf{f})$, and $(\mathbf{i}) \rho_{3}=3.5 \rho_{0}$.

\section{Discussion and conclusions}

In this work we have investigated the kinklike normal modes of a system of several loops with the help of the T-matrix theory. The results of this work can be summarized as follows:

1. In the system of two non-identical loops, we have found four kinklike normal modes $P_{x}, A P_{y}, P_{y}$, and $A P_{x}$. The frequencies of the $P_{x}$ and $A P_{y}$ solutions are very similar as well as the frequencies of the $P_{y}$ and $A P_{x}$ modes. This result agrees with Van Doorsselaere et al. (2008), who considered thin tubes (i.e. long wavelength approximation). For fat loops the $P_{x}$ and $A P_{y}$ modes, as well the $P_{y}$ and $A P_{x}$, have different frequencies, as was shown in Luna et al. (2008).

2. For a system of two loops we have investigated the dependence of the interaction between kink oscillations as a function on the relative density of the loop pair. For 

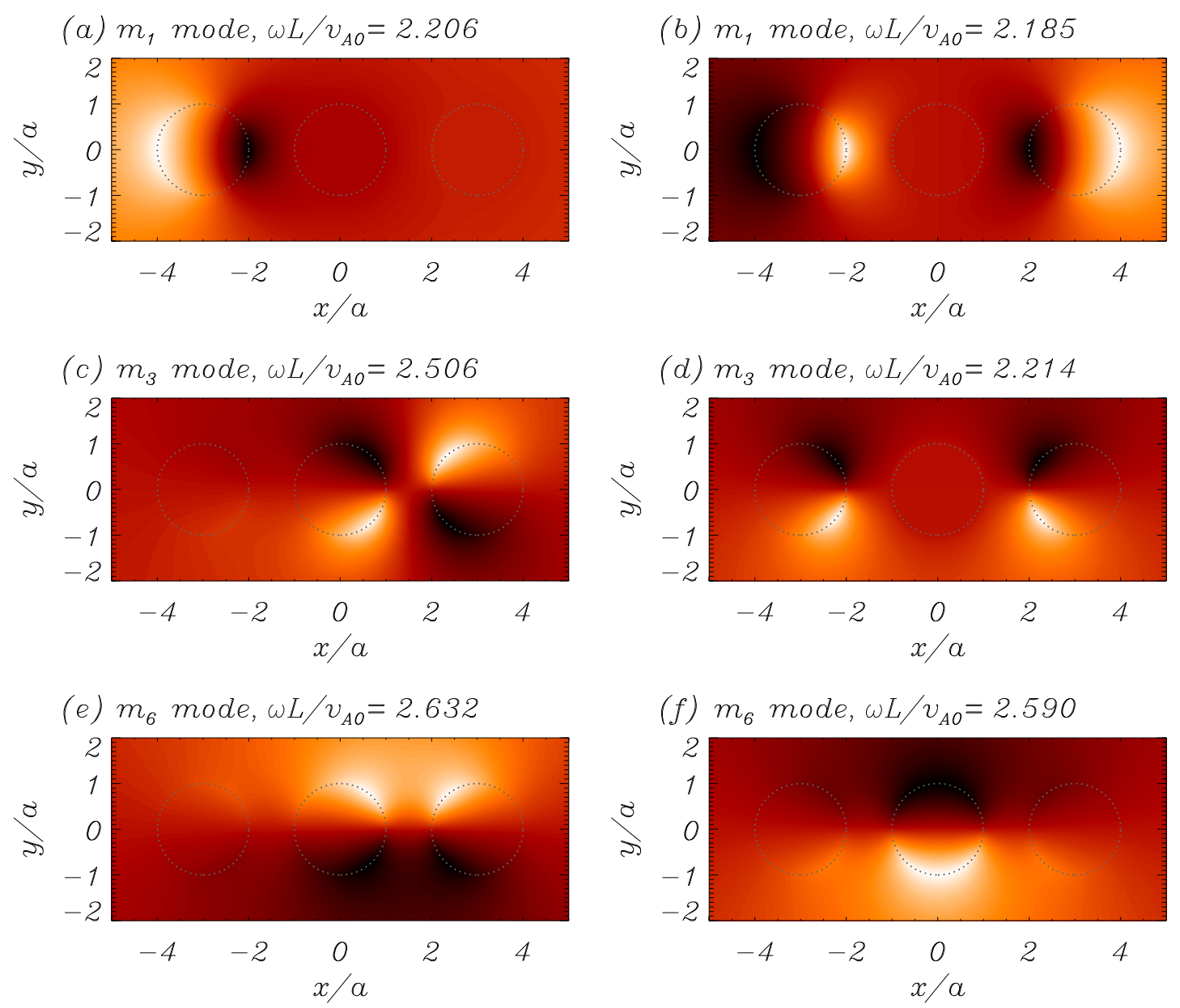

Fig. 7.- Same as Fig. 4 for two values of $\rho_{3}$ at the maximal coupling (Fig. 5). In the top, central, and bottom rows of panels the $m_{1}, m_{3}$, and $m_{6}$ modes are plotted for (a), (c), and (e) $\rho_{3}=2 \rho_{0} ;(\mathbf{b}),(\mathbf{d})$, and (f) $\rho_{3}=3 \rho_{0}$.

$\rho_{1}=3 \rho_{0}$ we have found that the oscillations of the loops are coupled in the range of $\rho_{2}$ between $2 \rho_{0}$ to $4 \rho_{0}$ and that the coupling is maximum at $\rho_{2}=\rho_{1}=3 \rho_{0}$. Outside this density range the loops are essentially decoupled and oscillate independently. This is qualitatively similar to the behavior of the anomalous modes described by Van Doorsselaere et al. (2008).

3. We have also studied the dependence of the interaction with the relative radii of the loops. We have seen that in the range of radii for which transverse loop oscillations have been observed the interaction depends very little on this parameter and the loops 
strongly interact for all the radii considered. The explanation of this behavior is that in our loops the thin loop approximation can be applied and in this situation the kink frequency depends on the tube density and not on the radii.

4. In the case of a system of three equal, aligned loops there are eight kinklike normal modes. The lower frequency mode corresponds to the three loops oscillating in phase in the $x$-direction, i.e. along the direction in which their axes are aligned, in agreement with the results of two identical loops. On the other hand, the upper frequency mode corresponds to the three loops oscillating in phase in the $y$-direction. This does not agree with the two identical loops situation, in which the upper mode corresponds to the two loops oscillating in antiphase in the $x$-direction. In fact, this property of the three-loop system is also true for ensembles of four or more aligned loops.

5. We have made a parametric study of the kinklike modes in a system of three loops with equal radii and different densities by changing the density of loop $3, \rho_{3}$. We have chosen $\rho_{1}=3 \rho_{0}$ and $\rho_{2}=2 \rho_{0}$ so that the interaction between loops 1 and 2 is negligible. We have found that the oscillations of loop 3 are coupled with loop 2 when $\rho_{3} \approx \rho_{2}$, whereas loop 1 oscillates independently. Furthermore, loop 3 couples with loop 1 when $\rho_{3} \approx \rho_{1}$ with loop 2 oscillating independently. If $\rho_{3}$ takes different values, the system is decoupled and the three loops oscillate independently.

In this work, we have found that the interaction between loops regarding kinklike motions depends strongly on the their individual kink frequencies. If these frequencies are similar, loop motions are coupled and the normal modes are collective. On the other hand, if the loop kink frequencies are quite different their motions are not coupled. Since the individual frequencies depend on the loop density and radius, we have studied separately the influence of the two parameters. We have found that if the densities are quite similar, loops are coupled and the oscillations are collective. On the other hand, if the densities are quite different, the tubes oscillate independently. The range of densities for which the loops are coupled depends on the system properties and in the configuration of three loops this range is narrower than in the two tubes configuration.

From the results shown in this paper we suggest that the antiphase motions reported in Schrijver and Brown (2000) and Schrijver et al. (2002) are collective motions and, therefore, that the individual kink frequencies are similar but different from the collective observed frequency. If the loop model presented here is valid, both loop densities are also similar. In addition, in Verwichte et al. (2004) a loop arcade is studied and three groups of tubes oscillating with similar frequencies can be appreciated. The dynamics of each group of tubes can be interpreted as collective, although a detailed study of such configuration is needed to 
relate the loop characteristics and the frequency of oscillation of the group. On the other hand, loops not belonging to these three groups do not share their frequencies with other loops, and so oscillate independently. This has to be interpreted as a sign that these loops have different densities from those of the rest of the loops. It must be mentioned that in Verwichte et al. (2004) all the oscillations are assumed as individual, but this is only true in the case of loops that do not share their frequency. If this assumption is applied to loop with a collective behavior it produces wrong results for the loop parameters. For example, if the loops actually oscillate with the lowest frequency collective mode, the assumption of these authors produces an underestimation of the magnetic field or an overestimation of the loop density.

The T-matrix method shown in this paper can be easily applied to more complex configurations with gas pressure and tubes with flows or more complex systems of loops, i.e. arcades with myriads of loops or multistranded loops. It is expected that in such systems loops oscillate essentially independently except for loops with similar individual oscillation frequencies.

We would like to thank Dr. Rony Keppens for calling our attention about the Tmatrix theory and Dr. A. J. Díaz for his comments. M. Luna is grateful to the Spanish Ministry of Science and Education for an FPI fellowship, which is partially supported by the European Social Fund. He also thanks the members of the Departament of Mathematics of K. U. Leuven for their warm hospitality during his brief stay at this University and for their worthy comments. The authors acknowledge the Spanish Ministry of Science and Education and the Conselleria d'Economia, Hisenda i Innovació of the Goverment of the Balearic Islands for the funding provided under grants AYA2006-07637, PRIB-2004-10145, and PCTIB-2005-GC3-03, respectively.

\section{REFERENCES}

Aschwanden, M. J., Fletcher, L., Schrijver, C. J., \& Alexander, D. 1999, ApJ, 520, 880

Aschwanden, M. J., De Pontieu, B., Schrijver, C. J., \& Title, A. M. 2002, Sol. Phys., 206, 99

Aschwanden, M. J., Nightingale, W., Andries, J., Goossens, M., \& Van Doorsselaere, T. 2003, ApJ, 598, 1375

Aschwanden, M. J., \& Nightingale, W. 2005, ApJ, 633, 499 
Berton, R., \& Heyvaerts, J. 1987, Sol. Phys., 109, 201

Bogdan, T. J., \& Zweibel, E. G. 1985, ApJ, 298, 867

Bogdan, T. J., \& Zweibel, E. G. 1987, ApJ, 312, 444

Bogdan, T. J., \& Cattaneo, F. 1989, ApJ, 342, 545

Bogdan, T. J. 1989, ApJ, 345, 1042

Bogdan, T. J., \& Kölner, M. 1991, ApJ, 369, 219

Bogdan, T. J., \& Fox, D. C. 1991, ApJ, 379, 758

Cally, P. S. 1986, Sol. Phys., 103, 277

Díaz, A. J., Oliver, R. \& Ballester, J. L., Roberts, B. 2004, A\&A, 424, 1055

Díaz, A. J., Oliver, R., \& Ballester, J. L. 2005, A\&A, 440, 1167

DeForest, C. E. 2007, ApJ, 661, 532

Edwin, P. M., \& Roberts, B. 1983, Sol. Phys., 88, 179

Goedbloed, J. P. 1983, Lecture Notes on Ideal Magnetohydrodynamics, Rijnhuizen Rep., 76

Keppens, R., Bogdan, T. M., \& Goossens, M. 1994, ApJ, 436, 372

Keppens, R. 1995, PHD Thesis, Katholieke Universiteit Leuven, Belgium

Klimchuk, J. A. 2006, Sol. Phys., 234, 41

Luna M., Terradas J., Oliver R., \& Ballester J. L., 2006, A\&A, 457, 1071

Luna M., Terradas J., Oliver R., \& Ballester J. L., 2008, ApJ, 676, 717

Murawski, K. 1993, Acta Astronomica, 43, 2, 161

Murawski, K., \& Roberts, B. 1994, Sol. Phys., 151, 305

Nakariakov, V. M., Ofman, L., DeLuca, E. E., Roberts, B., \& Davila, J. M. 1999, Science, 285, 862

Ramm, A. G. 1986, Scattering by obstacles (Dordrecht: Reidel)

Schrijver, C. J., \& Brown, D. S. 2000, ApJ, 537, L69 
Schrijver, C. J., Aschwanden, M. J., \& Title, A. M. 2002, Sol. Phys., 206, 69

Spruit, H. C. 1982, Sol. Phys., 75, 3

Terradas, J., Arregui, I., Oliver, R., Ballester, J. L., Andries, J., \& Goossens, M. 2008, ApJ, 679,1611

Tirry, W. J. 2000, ApJ, 528, 493

Van Doorsselaere, T., Ruderman, M. S., \& Robertson, D., 2008, A\&A, 485, 849

Verwichte, E., Nakariakov, V. M., Ofman, L., \& Deluca, E. E. 2004, Sol. Phys., 223, 77

Waterman, P. C., \& Truell, R. 1961, JMP, 2, 512

Waterman, P. C. 1969, ASAJ, 45, 1417 